\title{
Beta Linear Failure Rate Geometric Distribution with Applications
}

\author{
Broderick O. Oluyede ${ }^{1}$, Ibrahim Elbatal ${ }^{2}$, Shujiao Huang ${ }^{3}$ \\ ${ }^{I}$ Department of Mathematical Sciences, Georgia Southern University \\ ${ }^{2}$ Department of Mathematics and Statistics, Al Imam Mohammad Ibn Saud Islamic \\ University \\ ${ }^{3}$ Department of Mathematics, University of Houston
}

Abstract: This paper introduces the beta linear failure rate geometric (BLFRG) distribution, which contains a number of distributions including the exponentiated linear failure rate geometric, linear failure rate geometric, linear failure rate, exponential geometric, Rayleigh geometric, Rayleigh and exponential distributions as special cases. The model further generalizes the linear failure rate distribution. A comprehensive investigation of the model properties including moments, conditional moments, deviations, Lorenz and Bonferroni curves and entropy are presented. Estimates of model parameters are given. Real data examples are presented to illustrate the usefulness and applicability of the distribution.

Key words: Beta linear failure rate distribution, Linear failure rate geometric distribution, Moments, Maximum likelihood estimation.

\section{Introduction}

Let $G(x ; \phi)$ be the cumulative distribution function (cdf) of an absolutely continuous random variable $X$, where $\phi \in \Omega$ is the parameter vector. A general class generated from the logit of a beta random variable was introduced by Eugene et al. (2002). The cdf of the Beta-G distribution has the form

$$
\mathrm{F}(\mathrm{x} ; \mathrm{a}, \mathrm{b}, \phi)=\frac{B_{G(x ; \phi)}(a, b)}{\mathrm{B}(\mathrm{a}, \mathrm{b})}=I_{G(x ; \phi)}(a, b),
$$

where $\mathrm{a}>0$ and $\mathrm{b}>0$ are two additional parameters whose role is to introduce skewness and to vary tail weight, $\mathrm{G}(\mathrm{x} ; \phi)$ is an arbitrary parent/baseline cdf, $\mathrm{B}_{\mathrm{y}}(\mathrm{a}, \mathrm{b})=\int_{0}^{y} w^{a-1}(1-$ $w)^{b-1} d w$ is the incomplete beta function with $\mathrm{B}(\mathrm{a}, \mathrm{b})=\mathrm{B}_{1}(a, b)$ and $\mathrm{I}_{\mathrm{y}}(a, b)=\frac{B_{y}(a, b)}{B(a, b)}$ is the incomplete beta function ratio. One major benefit of this class of distributions is its ability of fitting skewed data that cannot be properly fitted by existing distributions. If $b=1$, we have $F(x)=[G(x)]^{a}$ which is called the exponentiated $G$ distribution (or the Lehmann type-I distribution). See for example, the exponentiated Weibull (Pal et al. (2006), Mudholkar et al. (1995)) and exponentiated exponential (Gupta and Kundu (1999)) distributions. Indeed, if $Z$ is a beta distributed random variable with parameters $a$ and $b$, then the cdf of $X=G^{-1}(Z)$ agrees with the cdf given in equation (1). As usual, a random variable $X$ with the cdf (1) is 
said to have a beta $-G$ (BG) distribution and will be denoted by $X \sim B G(a, b ; \phi)$. Some special cases of $B G$ distributions are given by Bidram et al. (2013).

The classes of distributions obtained when $a=1$ and $b=1$ in equation (1) are called, respectively, the frailty parameter and resilience parameter families with underlying distribution $G(x ; \phi)$ (See Marshall and Olkin (2007)). Clearly, for positive integer values of $b(a)$, the BG cdf with $a=1(b=1)$ is the cdf of a series (parallel) system with $b(a)$ independent components all having the $\operatorname{cdf} G(x ; \phi)$. Several well-known distributions that belong to the resilience parameter family include the exponentiated Weibull (EW) distribution (see Mudholkar et al. (1995), generalized (or exponentiated) exponential distribution proposed by Gupta and Kundu (1999), and exponentiated type distributions introduced by Nadarajah and Kotz (2006). The generalized exponential-geometric (GEG) distribution of Silva et al. (2010) also belongs to the resilience parameter family.

For general $a$ and $b$, we can express the cdf given in equation (1) in terms of the wellknown hypergeometric function, that is

$$
F(x ; a, b, \phi)=\frac{[G(x ; \phi)]^{a}}{a B(a, b)}{ }_{2} F_{1}(a, 1-b, a+1 ; G(x ; \phi)),
$$

where

$$
{ }_{2} F_{1}(\alpha, \theta, \gamma ; x)=\sum_{i=0}^{\infty} \frac{(\alpha)_{i}(\theta)_{i}}{(\gamma)_{i} i !} x^{i}, \quad \text { for }|x|<1,
$$

and $(\alpha)_{\mathrm{i}}=\frac{\Gamma(\alpha+i)}{\Gamma(\alpha)}=\alpha(\alpha+1) \ldots(\alpha+\mathrm{i}-1)$ denotes the ascending factorial of $\alpha$. We can obtain the properties of $F(x)$ for any beta- $G$ distribution defined from a parent $G(x ; \phi)$ distribution in equation (1) which, in principle, follow from the properties of the hypergeometric function that are well established in the literature; see, for example, section 9.1 of Gradshteyn and Ryzhik (2000). The probability density function (pdf) and hazard (failure) rate functions of a BG distribution corresponding to the cdf in equation (1) are given by

$$
\mathrm{f}(\mathrm{x} ; \mathrm{a}, \mathrm{b}, \phi)=\frac{\mathrm{g}(\mathrm{x} ; \phi)}{B(a, b)} G(x ; \phi)^{a-1}\{1-G(x ; \phi)\}^{b-1},
$$

and

$$
h(x ; a, b, \phi)=\frac{g(x ; \phi) G(x ; \phi)^{a-1}\{1-G(x ; \phi)\}^{b-1}}{B(a, b) I_{[1-G(x ; \phi)]}(b, a)},
$$

respectively, where $I_{[1-G(x ; \phi)]}(a, b)=1-I_{G(x ; \phi)}(a, b)=\bar{F}(x ; a, b, \phi)$ is the survival function of a BG distribution corresponding to the cdf in equation (1).

The beta Generalized (Beta-G) distribution (beta modified Weibull (BMW)) distribution introduced by Silva et al. (2010) is a rich class of generalized distributions. This class has captured considerable attention over the last few years. Sepanski and Kong (2007) applied the Beta- $\mathrm{G}$ distribution to model the size distribution of income. This distribution has been studied in the literature for various forms of the baseline distribution $G$. The beta-G distributions that 
have been explored include the beta normal (BN) (Eugene et al. (2002)), beta Fre $\mathrm{c}^{\prime}$ het (BFr) distribution (Nadarajah and Kotz (2004)), beta exponential (BE) distribution (Nadarajah and Kotz (2006)), the Beta Weibull (BW) distribution (Lee et al. (2007)), beta Weibull-geometric distribution (Cordeiro et al. (2011)), beta generalized half normal distribution (Pescim et al. (2010)), beta extended half-normal model (Cordeiro et al. (2014)), beta Burr XII distribution (Paranaíba et al. (2011)). Barreto-Souza et al. (2010) introduced the beta generalized exponential (BGE) distribution. The beta generalized Lindley distribution was presented by Oluyede and Yang (2015), and beta inverse Weibull (BIW) was developed by Khan (2010). Oluyede et al. (2015) introduced and studied the log generalized Lindley-Weibull distribution and applied the model to lifetime data. Recently, Cordeiro et al. (2011) introduced the betaWeibull geometric distribution in which $G(x ; \phi)$ in equation (1) was taken to be the cdf of the Weibull-geometric distribution of Barreto-Souza et al. (2011). Bidram (2012) proposed the beta exponential-geometric distribution, thereby extending the exponential-geometric distribution of Adamidis and Loukas (1998). Also, Bidram et al. (2013) introduced a new distribution that includes the Weibull-geometric distribution of Barreto-Souza et al. (2010). Percontini et al. (2013) introduced and discussed the properties of the beta Weibull Poisson distribution. Cordeiro et al. (2014) presented the properties of Kummer beta generalized gamma distribution.

In this article, we attempt to generalized the linear failure rate geometric (LFRG) distribution of Mahmoudi and Jafari (2014) by taking $G(x ; \phi)$ in equation (1) to be the cdf of a

LFRG distribution, when $C(\theta)=\frac{\theta}{1-\theta}, 0<\theta<1$. Mahmoudi and Jafari (2014) compounded the linear failure rate distribution with a geometric distribution to obtained new lifetime distribution with five possible shapes for the hazard rate function, that is, increasing, decreasing, upside - down bathtub (unimodal), bathtub and increasing - decreasing - increasing shaped, which are common in reliability and biological studies. Recent generalizations of the linear failure rate distributions include the gamma linear failure rate distribution (Cordeiro et al. (2014)), and the Poisson generalized linear failure rate model (Cordeiro et al. (2015)).

The reminder of the paper is organized as follows. In Section 2, we define the beta linear failure rate geometric distribution, expansion for the cumulative and density functions, hazard and reverse hazard functions and some special cases are presented. Moments, moment generating function and conditional moments are discussed in Section 3. In Section 4, we obtain the mean deviations about the mean and the median, Bonferroni and Lorenz curves. Section 5 contains the distribution of the order statistics and uncertainty measures including Rényi and s-entropies. Maximum likelihood estimation is performed in Section 6. Applications are given in section 7, followed by concluding remarks.

\section{Beta Linear Failure Rate Geometric Distribution}

Consider the linear failure rate geometric (LFRG) distribution of Mahmoudi and Jafari (2014) with the cdf 


$$
G(x ; \alpha, \beta, \theta)=\frac{1-e^{-\left(\alpha x+\frac{\beta}{2} x^{2}\right)}}{1-\theta e^{-\left(\alpha x+\frac{\beta}{2} x^{2}\right)}}, \quad x>0,
$$

where $\alpha>0, \beta>0$, and $\theta \in(0,1)$. Replacing $G(x ; \phi)$, where $\phi=(\alpha, \beta, \theta)$ in equation (1) by the LFRG cdf in equation (4) above yields a new cdf

$$
\begin{aligned}
F_{B L F R G}(x ; \alpha, \beta, \theta, a, b) & =\frac{1}{B(a, b)} \int_{0-\theta e^{-\left(\alpha x+\frac{\beta}{2} x^{2}\right)}}^{1-w^{-\left(\alpha x+\frac{\beta}{2} x^{2}\right)}}(1-w)^{b-1} d w \\
& =I_{G(x ; \alpha, \beta, \theta)}(a, b)
\end{aligned}
$$

for $x>0, a>0, b>0, \alpha>0, \beta>0$, and $\theta \in(0,1)$. A random variable $X$ with the cdf in equation (0.5) is said to have a beta linear failure rate geometric (BLFRG) distribution and will be denoted by $X: B L F R G(a, b, \alpha, \beta, \theta)$. The corresponding pdf of this new distribution is given by

$$
f_{B L F R G}(x ; \alpha, \beta, \theta, a, b)=\frac{(1-\theta)^{b}(\alpha+\beta x) e^{-b\left(\alpha x+\frac{\beta}{2} x^{2}\right)}\left(1-e^{-\left(\alpha x+\frac{\beta}{2} x^{2}\right)}\right)^{a-1}}{B(a, b)\left(1-\theta e^{-\left(\alpha x+\frac{\beta}{2} x^{2}\right)}\right)^{a+b}},
$$

for $x>0$, where the parameters $a>0$ and $b>0$ are shape parameters, which characterize the skewness, kurtosis, and unimodality of the distribution.

Graphs of the pdf of BLFRG distribution are given in the Figure 1. The plots show that the BLFR pdf can be decreasing or right skewed among several other possible shapes as seen in the figure. The distribution has positive asymmetry. 

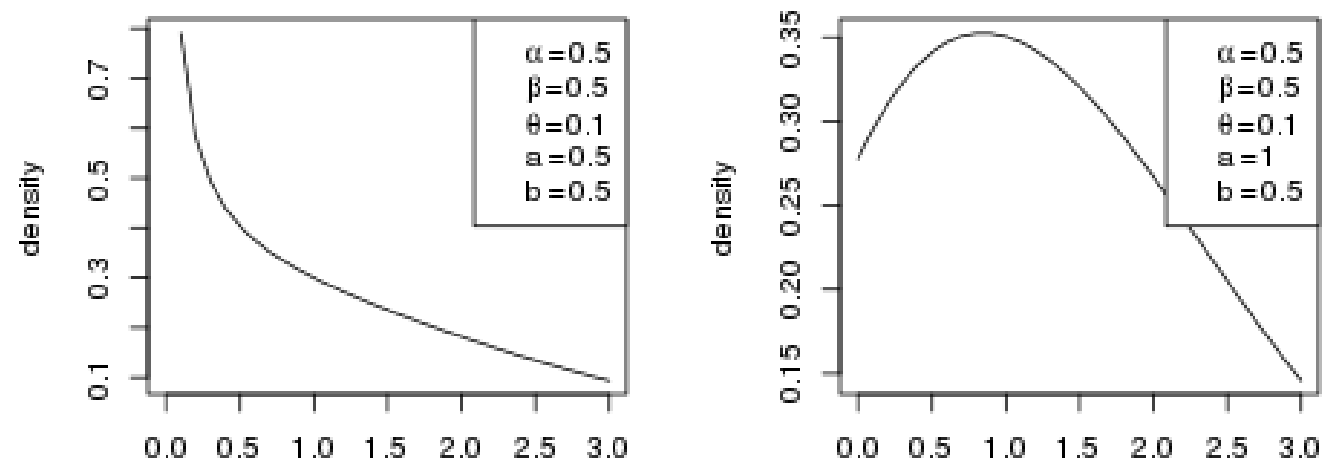

$x$

$\mathrm{x}$
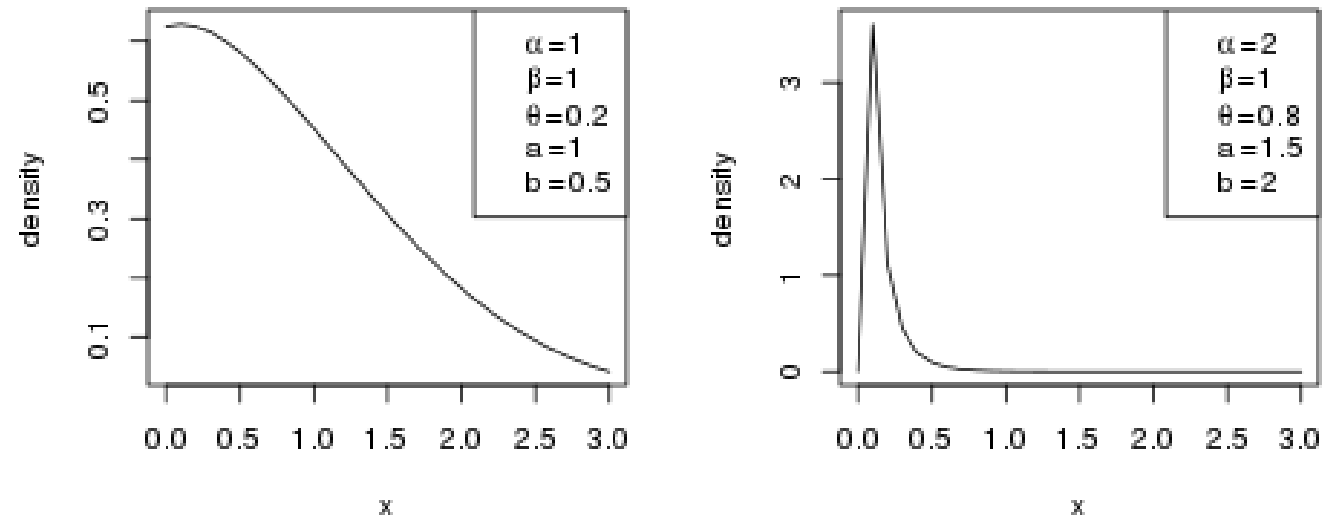

Figure 1: Graphs of BLFRG pdf for Selected Parameters

The reliability (survival) function (RF) of the BLFRG distribution, denoted by $R_{B L F R G}(x)$ is given by

$$
R_{B L F R G}(x)=1-F_{B L F R G}(x)=I_{\{1-G(x ; \alpha, \beta, \theta)\}}(b, a) .
$$

The hazard and reverse hazard functions of the BLFRG distribution are given by

$$
h_{B L F R G}(x)=\frac{f_{B L F R G}(x)}{R_{B L F R G}(x)}
$$




$$
=\frac{(1-\theta)^{b}(\alpha+\beta x) e^{-b\left(\alpha x+\frac{\beta}{2} x^{2}\right)}\left(1-e^{-\left(\alpha x+\frac{\beta}{2} x^{2}\right)}\right)^{a-1}}{B(a, b)\left(1-\theta e^{-\left(\alpha x+\frac{\beta}{2} x^{2}\right)}\right)^{a+b} I_{\{1-G(x ; \alpha, \beta, \theta)\}}(b, a)}
$$

and

$$
\begin{aligned}
\tau_{B L F R G}(x)= & \frac{f_{\text {BLFRG }}(x)}{F_{\text {BLFRG }}(x)} \\
= & \frac{(1-\theta)^{b}(\alpha+\beta x) e^{-b\left(\alpha x+\frac{\beta}{2} x^{2}\right)}\left(1-e^{-\left(\alpha x+\frac{\beta}{2} x^{2}\right)}\right)^{a-1}}{B(a, b)\left(1-\theta e^{-\left(\alpha x+\frac{\beta}{2} x^{2}\right)}\right)^{a+b} I_{\{G(x ; \alpha, \beta, \theta)\}}(a, b)},
\end{aligned}
$$

respectively. The density and hazard functions can exhibit different behavior depending on the values of the parameters when chosen to be positive, as shown in these plots. However, it is hard to analyze the shape of both the density and hazard function due to their complicated forms. Plots of the hazard rate function for different combinations of the parameter values are given in Figure 2. The plot shows various shapes including monotonically increasing, and bathtub shapes for five combinations of the values of the parameters. This flexibility makes the BLFRG hazard rate function suitable for both monotonic and non-monotonic empirical hazard behaviors that are likely to be encountered in real life situations. 

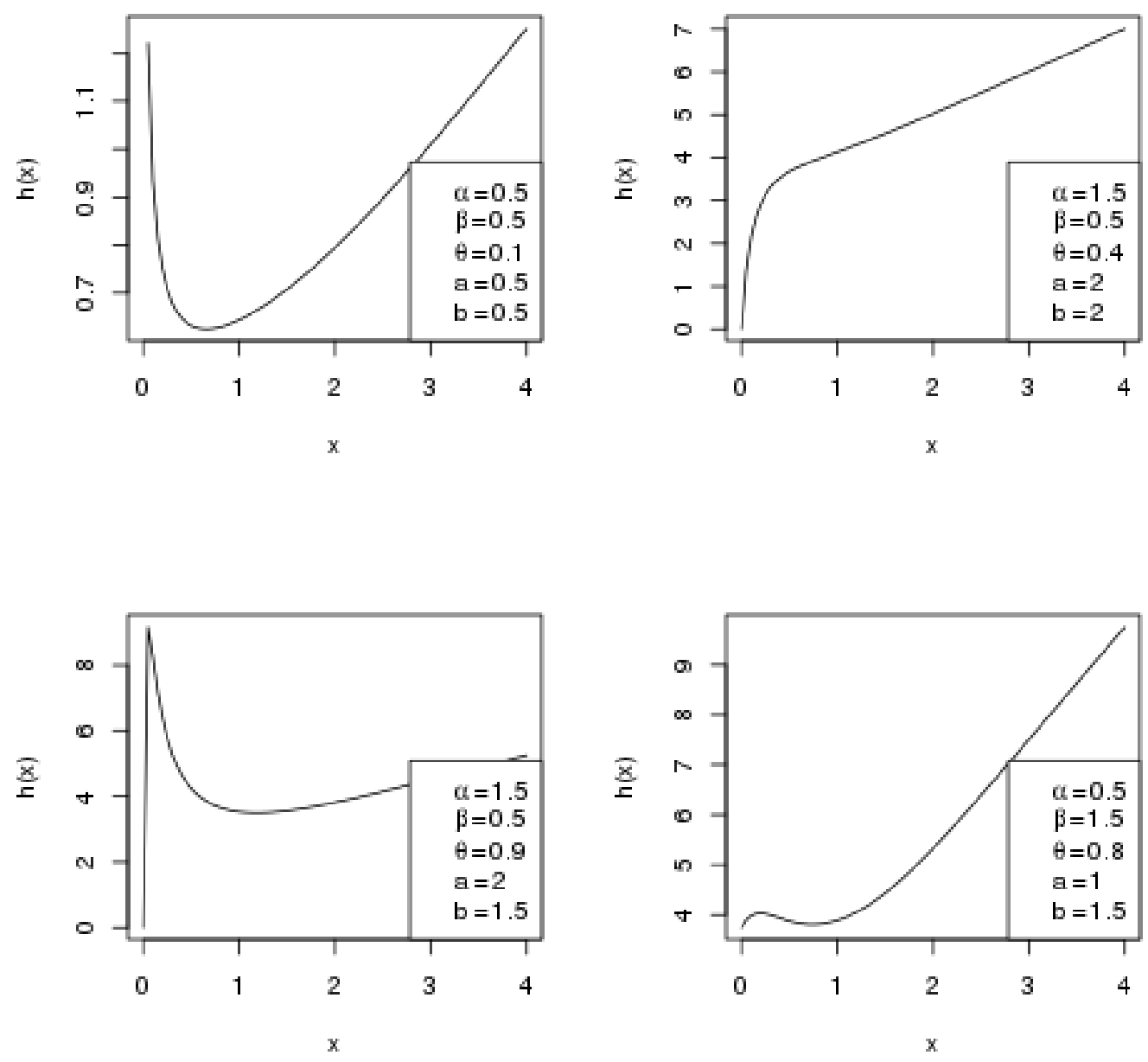

Figure 2: Graphs of BLFRG Hazard Function for Selected Parameters

\subsection{Special Cases of the BLFRG Distribution}

The BLFRG distribution is a very flexible model that approaches different distributions when its parameters are changed. The BLFRG distribution contains several special-models including the following distributions.

- If $\beta=0$, the $B L F R G$ distribution reduces to the lifetime distribution called beta exponential geometric (BEG) distribution which was proposed by Bidram (2012).

- The exponential geometric (EG) distribution is obtained when $a=b=1$, and $\beta=0$. If in addition, $\theta \downarrow 0$, the exponential distribution with parameter $\alpha>0$ is obtained. 
- When $a=b=1$, the BLFRG distribution reduces to the linear failure rate geometric (LFRG) distribution.

- When $\theta \downarrow 0$ we get beta linear failure rate (BLFR) distribution which was introduced by Mahmoudi and Jafari (2014).

- Generalized linear failure rate (GLFR) distribution arises when $\theta \downarrow 0$ and $b=1$ which was given by Sarhan and Kundu (2009). In addition, when $a=1$, the linear failure rate (LFR) distribution is obtained.

- When $\alpha=0$, we get the beta Rayleigh geometric (BRG) distribution. In addition, when $\theta \downarrow 0$, the beta Rayleigh (BR) distribution is obtained.

- $\quad$ Rayleigh geometric (RG) distribution arises as a special case of BLFRG by taking $\alpha=0$ and $a=b=1$.

- When $\beta=0$, and $\theta \downarrow 0$, we obtain the beta exponential (BE) distribution.

- $\quad$ Rayleigh distribution is obtained when $\alpha=0, \theta \downarrow 0$, and $a=b=1$.

- Exponentiated linear failure rate geometric distribution (ELFRG) is obtained when $b=1$.

\subsection{Quantile Function}

In this subsection, we present the quantile function of the BLFRG distribution. The quantile of the BLFRG distribution is obtained by solving the nonlinear equation $I_{\{G(x ; \alpha, \beta, \theta)\}}(a, b)=U$, where $G(x ; \alpha, \beta, \theta)=\left(1-e^{-\left(\alpha x+\frac{\beta}{2} x^{2}\right)}\right) /\left(1-\theta e^{-\left(\alpha x+\frac{\beta}{2} x^{2}\right)}\right)$, and $U$ is a uniform variate on the unit interval $[0,1]$. It follows that the BLFRG variate $X$ are the roots of the equation

$$
\beta X^{2}+2 \alpha X+2 \log \left(\frac{1-I_{u}^{-1}(a, b)}{1-\theta I_{u}^{-1}(a, b)}\right)=0
$$

that is

$$
X=\frac{-\alpha+\sqrt{\alpha^{2}-2 \beta \log \left(\frac{1-I_{u}^{-1}(a, b)}{1-\theta I_{u}^{-1}(a, b)}\right)}}{\beta},
$$

for $\alpha, \beta>0$, and $X=\frac{-\log \left(\frac{1-I_{u}^{-1}(a, b)}{1-\theta I_{u}^{-1}(a, b)}\right)}{\alpha}$, if $\beta=0$ and $\alpha>0$, where $I_{u}^{-1}(a, b)$

denotes the inverse of the incomplete beta function ratio. Quantiles of the BLFRG distribution for selected values of the model parameters are given in Table 1 . An $\mathrm{R}$ algorithm for the computation of the quantiles of the BLFRG distribution is given in the appendix. 
Table 1 BLFRG Quantiles for Selected Parameter Values

\begin{tabular}{c|cccc}
\hline & \multicolumn{4}{|c}{$(\alpha, \beta, \theta, \mathrm{a}, \mathrm{b})$} \\
\hline$u$ & $(3,5,0.5,4,9)$ & $(5,2,0.1,9,4)$ & $(15,1,0.8,10,2)$ & $(1,3,0.2,8,6)$ \\
\hline 0.1 & 0.02840435 & 0.1343841 & 0.02450469 & 0.2973634 \\
0.2 & 0.03760774 & 0.159947 & 0.0314877 & 0.3435863 \\
0.3 & 0.04546788 & 0.1804346 & 0.03775505 & 0.3790015 \\
0.4 & 0.05306507 & 0.1993744 & 0.04410258 & 0.4106138 \\
0.5 & 0.06094589 & 0.2183185 & 0.05099674 & 0.4412642 \\
0.6 & 0.06962035 & 0.2385029 & 0.05894946 & 0.4729599 \\
0.7 & 0.07982285 & 0.2615153 & 0.06878013 & 0.5080052 \\
0.8 & 0.09303174 & 0.2903687 & 0.08223558 & 0.5504758 \\
0.9 & 0.11373300 & 0.3339392 & 0.10482113 & 0.6119009 \\
\hline
\end{tabular}

\subsection{Expansion for the Cumulative and Density Functions}

In this subsection, we present some series representations of the cdf and pdf of the BLFRG distribution. The mathematical relation given below will be useful in subsequent sections. Here and henceforth, we let $X$ be a random variable having the $\operatorname{BLFRG}(a, b, \alpha, \beta, \theta)$ distribution. We obtain some alternative expressions for the cdf $F_{B L F R G}(x)$ and pdf $f_{B L F R G}(x)$ of the BLFRG distribution. The following series representations will be useful in studying the properties of the BLFRG distribution. If $b$ is a positive real non-integer and $|z|<1$, then

$$
(1-z)^{b-1}=\sum_{j=0}^{\infty} \frac{(-1)^{j} \Gamma(b)}{\Gamma(b-j) j !} z^{j} .
$$

Applying the series representation, one can re-write the BLFRG cdf as follows:

$$
\begin{aligned}
F_{B L F R G}(x ; \alpha, \beta, \theta, a, b) & =\frac{\Gamma(a+b)}{\Gamma(a)} \sum_{j=0}^{\infty} \frac{(-1)^{j}}{\Gamma(b-j) j !(a+j)}[G(x ; \alpha, \beta, \theta)]^{a+j} \\
& =\frac{\Gamma(a+b)}{\Gamma(a)} \sum_{j=0}^{\infty} \frac{(-1)^{j} F_{G L F R G}(x ; \alpha, \beta, \theta, a+j)}{\Gamma(b-j) j !(a+j)}
\end{aligned}
$$

Consequently, the BLFRG cdf can be expressed as a mixture of generalized linear failure rate geometric (GLFRG) distributions with parameters $\alpha, \beta, \theta$ and $a+j$. Also, when $b>0$ is an integer the index $j$ in the series representation stops at $b-1$. Using the series representation below:

$$
(1-z)^{-k}=\sum_{j=0}^{\infty} \frac{\Gamma(k+j)}{\Gamma(k) j !} z^{j}, \quad|z|<1, \text { and } k>0,
$$


the BLFRG pdf can be expressed as

$$
\begin{aligned}
f_{B L F R G}(x ; \alpha, \beta, \theta, a, b) & =\frac{(1-\theta)^{b}}{\Gamma(b)} \sum_{j=0}^{\infty} \frac{\Gamma(b+j) \theta^{j}}{j ! B(a, b+j)}(\alpha+\beta x) e^{-(b+j)\left(\alpha x+\frac{\beta}{2} x^{2}\right)} \\
& \times\left(1-e^{-\left(\alpha x+\frac{\beta}{2} x^{2}\right)}\right)^{a-1} \\
& =\frac{(1-\theta)^{b}}{\Gamma(b)} \sum_{j=0}^{\infty} \frac{\Gamma(b+j) \theta^{j} f_{B L F R}(x ; \alpha, \beta, a, b+j)}{j ! B(a, b+j)}
\end{aligned}
$$

where $f_{B L F R}(x ; \alpha, \beta, \theta, a, b+j)$ is the pdf of the BLFR distribution that was introduced by Mahmoudi and Jafari (2014). Another form of equation (0.15) is as follows:

$$
\begin{aligned}
f_{B L F R G}(x ; \alpha, \beta, \theta, a, b) & =\frac{(1-\theta)^{b}}{\Gamma(b)} \sum_{j=0}^{\infty} \frac{\Gamma(b+j) \theta^{j}}{j ! B(a, b+j)}(\alpha+\beta x) e^{-(b+j)\left(\alpha x+\frac{\beta}{2} x^{2}\right)} \\
& \times\left(1-e^{-\left(\alpha x+\frac{\beta}{2} x^{2}\right)}\right)^{a-1} \\
& =\frac{(1-\theta)^{b}}{\Gamma(b)} \sum_{i=0}^{\infty} \sum_{j=0}^{\infty} \frac{\Gamma(b+j) \theta^{j}(-1)^{i}}{j ! B(a, b+j)}\left(\begin{array}{c}
a-1 \\
i
\end{array}\right) \\
& \times(\alpha+\beta x) e^{-(b+i+j)\left(\alpha x+\frac{\beta}{2} x^{2}\right)}
\end{aligned}
$$

Now, since $0<e^{-\left(\alpha x+\frac{\beta}{2} x^{2}\right)}<1$, and using Taylor expansion of the function $e^{-(b+i+j)\left(\frac{\beta}{2} x^{2}\right)}$ given by

$$
e^{-(b+i+j)\left(\frac{\beta}{2} x^{2}\right)}=\sum_{k=0}^{\infty} \frac{(-1)^{k}\left[(b+i+j)\left(\frac{\beta}{2}\right)\right]^{k} x^{2 k}}{k !},
$$

equation (16) becomes

$$
f_{B L F R G}(x ; \alpha, \beta, \theta, a, b)=\sum_{i, j, k=0}^{\infty} \frac{w_{i, j, k}(1-\theta)^{b}}{k ! \Gamma(b)} x^{2 k}(\alpha+\beta x) e^{-(b+i+j)(\alpha x)},
$$

where

$$
w_{i, j, k}=\frac{\Gamma(b+j) \theta^{j}(-1)^{i+k}\left(\begin{array}{c}
a-1 \\
i
\end{array}\right)}{B(a, b+j) j ! k !}\left[(b+i+j)\left(\frac{\beta}{2}\right)\right]^{k} .
$$

\section{Moments and Conditional Moments}


In this section, we present the moments and conditional moments of the BLFRG distribution. Moments are necessary and important in any statistical analysis, especially in applications. It can be used to study the most important features and characteristics of a distribution (e.g., tendency, dispersion, skewness and kurtosis). A table of the first six moments and related statistics for selected values of the model parameters is also presented.

\subsection{Moments}

If $X$ has the BLFRG distribution, then the $r^{\text {th }}$ moment of $X$ is given by

$$
\mu_{r}=\sum_{i, j, k=0}^{\infty} \frac{w_{i, j, k}(1-\theta)^{b}}{k ! \Gamma(b)}\left[\frac{\Gamma(r+2 k+1)}{[\alpha(b+i+j)]^{r+2 k+1}}\left(\alpha+\frac{\beta(r+2 k+1)}{\alpha(b+i+j)}\right)\right] .
$$

Note that

$$
\begin{aligned}
\mu_{r} & =\sum_{i, j, k=0}^{\infty} \frac{w_{i, j, k}(1-\theta)^{b}}{k ! \Gamma(b)} \int_{0}^{\infty} x^{r+2 k}(\alpha+\beta x) e^{-(b+i+j)(\alpha x)} d x \\
& =\sum_{i, j, k=0}^{\infty} \frac{w_{i, j, k}(1-\theta)^{b}}{k ! \Gamma(b)}\left[\frac{\Gamma(r+2 k+1)}{[\alpha(b+i+j)]^{r+2 k+1}}\left(\alpha+\frac{\beta(r+2 k+1)}{\alpha(b+i+j)}\right)\right] .
\end{aligned}
$$

The coefficients of variation, skewness and kurtosis of the BLFRG distribution can be readily obtained according to the following relation $\mathrm{SD}_{B L F R G}=\sqrt{\mu_{2}-\mu_{1}^{1}}, \mathrm{CV}_{B L F R G}=\sqrt{\frac{\mu_{2}}{\mu_{1}^{2}}-1}$, $\mathrm{CS}_{B L F R G}=\frac{\mu_{3}-3 \mu_{1} \mu_{2}+2 \mu_{1}^{3}}{\left[\mu_{2}-\mu_{1}^{2}\right]^{\frac{3}{2}}}$ and $\mathrm{CK}_{B L F R G}=\frac{\mu_{4}-4 \mu_{1} \mu_{3}+6 \mu_{1}^{2} \mu_{2}-3 \mu_{1}^{4}}{\left[\mu_{2}-\mu_{1}^{2}\right]^{2}}$.

Table 2 Table of Moments and Related Statistics

\begin{tabular}{c|cccc}
\hline & \multicolumn{4}{|c}{$(\alpha, \beta, \theta, \mathrm{a}, \mathrm{b})$} \\
\hline & $(0.5,0.5,0.1,0.5,0.5)$ & $(0.5,0.5,0.1,1.0,0.5)$ & $(1.0,1.0,0.2,1.0,0.5)$ & $(2.0,1.0,0.8,1.5,2.0)$ \\
\hline $\mathrm{EX}$ & 1.2255612 & 1.7034846 & 1.0236743 & 0.101120946 \\
$\mathrm{EX}^{2}$ & 2.8861090 & 4.3150440 & 1.6618322 & 0.022906403 \\
$\mathrm{EX}^{3}$ & 8.8818292 & 13.6401956 & 3.4547881 & 0.008988826 \\
$\mathrm{EX}^{4}$ & 32.3777643 & 50.3041555 & 8.4920167 & 0.005148664 \\
$\mathrm{EX}^{5}$ & 133.6732315 & 208.8162045 & 23.6857641 & 0.003846790 \\
$\mathrm{EX}^{6}$ & 609.1805267 & 954.1735886 & 73.1189732 & 0.003491977 \\
$\mathrm{SD}$ & 1.1764815 & 1.1887742 & 0.7835325 & 0.112609757 \\
$\mathrm{CV}$ & 0.9599533 & 0.6978485 & 0.7654118 & 1.113614554
\end{tabular}




\subsection{Conditional Moments}

For lifetime models, it is also of interest to find the conditional moments and the mean residual lifetime function. The $n^{\text {th }}$ conditional moments for BLFRG distribution is given by

$$
\begin{aligned}
E\left(X^{n} \mid X>t\right) & =\frac{1}{\bar{F}_{B L F R G}(x)} \int_{t}^{\infty} x^{n} f_{B L F R G}(x) d x \\
& =\sum_{i, j, k=0}^{\infty} \frac{w_{i, j, k}(1-\theta)^{b}}{k ! \Gamma(b) \bar{F}_{B L F R G}(x)} \int_{t}^{\infty} x^{2 k+n}(\alpha+\beta x) e^{-(b+i+j)(\alpha x)} d x \\
& =\sum_{i, j, k=0}^{\infty} \frac{w_{i, j, k}(1-\theta)^{b}}{k ! \Gamma(b) \bar{F}_{B L F R G}(x)[\alpha(b+i+j)]^{2 k+n+1}} \\
& \times(\alpha \Gamma(2 k+n+1, \alpha(b+i+j) t) \\
& +\beta[\alpha(b+i+j)] \Gamma(2 k+n+2, \alpha(b+i+j) t)),
\end{aligned}
$$

where $\Gamma(s, t)=\int_{t}^{\infty} x^{s-1} e^{-x} d x$ is the upper incomplete gamma function. The mean residual lifetime function is given by

$$
\begin{aligned}
E(X \mid X>t)-t & =\sum_{i, j, k=0}^{\infty} \frac{w_{i, j, k}(1-\theta)^{b}}{k ! \Gamma(b) \bar{F}_{B L F R G}(x)[\alpha(b+i+j)]^{2 k+2}} \\
& \times(\alpha \Gamma(2 k+2, \alpha(b+i+j) t) \\
& +\beta[\alpha(b+i+j)] \Gamma(2 k+3, \alpha(b+i+j) t))-t .
\end{aligned}
$$

\section{Mean Deviations, Bonferroni and Lorenz Curves}

In this section we obtain mean deviation about the mean and the mean deviation about the median as well as Bonferroni and Lorenz curves for the BLFRG distribution.

\subsection{Mean Deviations}

The amount of scatter in a population is evidently measured to some extent by the totality of deviations from the mean and median. These are known as the mean deviation about the mean and the mean deviation about the median. They are defined by

$$
\delta_{1}(x)=\int_{0}^{\infty}|x-\mu| f(x) d x \quad \text { and } \quad \delta_{2}(x)=\int_{0}^{\infty}|x-M| f(x) d x,
$$


respectively, where $\mu=E(X)$ and $M=\operatorname{Median}(X)$ denotes the median. The measures $\delta_{1}(x)$ and $\delta_{2}(x)$ can be calculated using the relationships

$$
\delta_{1}(x)=2 \mu F(\mu)-2 \mu+2 \int_{\mu}^{\infty} x f(x) d x,
$$

and

$$
\delta_{2}(x)=-\mu+2 \int_{M}^{\infty} x f(x) d x
$$

respectively. When $r=1$ in equation (??), we get the mean $\mu=E(X)$ as

$$
\mu=\sum_{i, j, k=0}^{\infty} \frac{w_{i, j, k}(1-\theta)^{b}}{k ! \Gamma(b)}\left[\frac{\Gamma(2 k+2)}{[\alpha(b+i+j)]^{2 k+2}}\left(\alpha+\frac{\beta(2 k+2)}{\alpha(b+i+j)}\right)\right] .
$$

Note that $\int_{\mu}^{\infty} x f_{B L F R G}(x) d x$ and $\int_{M}^{\infty} x f_{B L F R G}(x) d x$ are given by

$$
\begin{aligned}
\int_{\mu}^{\infty} x f_{B L F R G}(x) d x & =\sum_{i, j, k=0}^{\infty} \frac{w_{i, j, k}(1-\theta)^{b}}{k ! \Gamma(b)[\alpha(b+i+j)]^{2 k+2}}(\alpha \Gamma(2 k+2, \alpha(b+i+j) \mu) \\
& +\beta[\alpha(b+i+j)] \Gamma(2 k+3, \alpha(b+i+j) \mu))
\end{aligned}
$$

and

$$
\begin{aligned}
\int_{M}^{\infty} x f_{B L F R G}(x) d x & =\sum_{i, j, k=0}^{\infty} \frac{w_{i, j, k}(1-\theta)^{b}}{k ! \Gamma(b)[\alpha(b+i+j)]^{2 k+2}}(\alpha \Gamma(2 k+2, \alpha(b+i+j) M) \\
& +\beta[\alpha(b+i+j)] \Gamma(2 k+3, \alpha(b+i+j) M)),
\end{aligned}
$$

respectively, so that the mean deviation about the mean is

$$
\begin{aligned}
\delta_{1}(x) & =2 \mu F_{B L F R G}(\mu)-2 \mu+2 \sum_{i, j, k=0}^{\infty} \frac{w_{i, j, k}(1-\theta)^{b}}{k ! \Gamma(b)[\alpha(b+i+j)]^{2 k+2}} \\
& \times(\alpha \Gamma(2 k+2, \alpha(b+i+j) \mu) \beta[\alpha(b+i+j)] \Gamma(2 k+3, \alpha(b+i+j) \mu))
\end{aligned}
$$

and the mean deviation about the median is

$$
\begin{gathered}
\delta_{2}(x)=-\mu+2 \sum_{i, j, k=0}^{\infty} \frac{w_{i, j, k}(1-\theta)^{b}}{k ! \Gamma(b)[\alpha(b+i+j)]^{2 k+2}}(\alpha \Gamma(2 k+2, \alpha(b+i+j) M) \\
+\beta[\alpha(b+i+j)] \Gamma(2 k+3, \alpha(b+i+j) M)) .
\end{gathered}
$$

\subsection{Bonferroni and Lorenz Curves}

In this subsection, we present Bonferroni and Lorenz Curves. Bonferroni and Lorenz curves (Bonferroni (1930)) have applications not only in economics for the study income and poverty, but also in other fields such as reliability, demography, insurance and medicine. Bonferroni and Lorenz curves are given by 


$$
\begin{aligned}
B(p) & =\frac{1}{p \mu} \int_{0}^{q} x f_{B L F R G}(x) d x \\
& =\frac{1}{p \mu} \sum_{i, j, k=0}^{\infty} \frac{w_{i, j, k}(1-\theta)^{b}}{k ! \Gamma(b)[\alpha(b+i+j)]^{2 k+2}}(\alpha \gamma(2 k+2, \alpha(b+i+j) q) \\
& +\beta[\alpha(b+i+j)] \gamma(2 k+3, \alpha(b+i+j) q)),
\end{aligned}
$$

and

$$
\begin{aligned}
L(p) & =\frac{1}{\mu} \int_{0}^{q} x f_{B L F R G}(x) d x \\
& =\frac{1}{\mu} \sum_{i, j, k=0}^{\infty} \frac{w_{i, j, k}(1-\theta)^{b}}{k ! \Gamma(b)[\alpha(b+i+j)]^{2 k+2}}(\alpha \gamma(2 k+2, \alpha(b+i+j) q) \\
& +\beta[\alpha(b+i+j)] \gamma(2 k+3, \alpha(b+i+j) q)),
\end{aligned}
$$

respectively, where $\gamma(\alpha, b)=\int_{0}^{b} t^{\alpha-1} e^{-t} d t$ is the lower incomplete gamma function.

\section{Order Statistics and Measures of Uncertainty}

In this section, the distribution of order statistics and measures of uncertainty for the BLFRG distribution are presented. The concept of entropy plays a vital role in information theory. The entropy of a random variable is defined in terms of its probability distribution and can be shown to be a good measure of randomness or uncertainty.

\subsection{Distribution of Order Statistics}

Suppose that $X_{1}, \cdots, X_{n}$ is a random sample of size $n$ from a continuous pdf, $f(x)$. Let $X_{1: n}<X_{2: n}<\cdots<X_{n: n}$ denote the corresponding order statistics. If $X_{1}, \cdots, X_{n}$ is a random sample from BLFRG distribution, it follows from the equations $(0.5)$ and $(0.6)$ that the pdf of the $k^{\text {th }}$ order statistics, say $Y_{k}=X_{k: n}$ is given by

$$
\begin{aligned}
f_{k}\left(y_{k}\right) & =\frac{n !}{(k-1) !(n-k) !} \sum_{l=0}^{n-k} n-k \quad(-1)^{l}\left(\frac{B_{G\left(y_{k} ; \alpha, \beta, \theta\right)}(a, b)}{B(a, b)}\right)^{k-1+l} \\
& \times \frac{(1-\theta)^{b}}{B(a, b)}\left(\alpha+\beta y_{k}\right) e^{-b\left(\alpha y_{k}+\frac{\beta}{2} y_{k}^{2}\right)} \sum_{i=0}^{\infty}\left(\begin{array}{c}
a-1 \\
i
\end{array}\right)(-1)^{1} e^{-i\left(\alpha y_{k}+\frac{\beta}{2} y_{k}^{2}\right)}
\end{aligned}
$$




$$
\begin{aligned}
& \times \sum_{j=0}^{\infty} \frac{\Gamma(a+b+j) \theta^{j}}{\Gamma(j+1) \Gamma(a+b)} e^{-j\left(\alpha y_{k}+\frac{\beta}{2} y_{k}^{2}\right)} \\
& =\sum_{l=0}^{n-k} \sum_{i, j=0}^{\infty} \frac{n !(1-\theta)^{b} \theta^{j}\left[B_{G\left(y_{k} ; \alpha, \beta, \theta\right)}\right]^{k+l-1}}{(k-1) !(n-k) ![B(a, b)]^{k+l}(b+i+j)} \\
& \times(b+i+j)\left(\alpha+\beta y_{k}\right) e^{-(b+i+j)\left(\alpha y_{k}+\frac{\beta}{2} y_{k}^{2}\right)}
\end{aligned}
$$

The corresponding cdf of $Y_{k}$ is

$$
\begin{aligned}
& F_{k}\left(y_{k}\right)=\sum_{j=k}^{n} \sum_{l=0}^{n-j} n \quad n-j \quad(-1)^{l}\left[F\left(y_{k}\right)\right]^{j+l} \\
& =\sum_{j=k}^{n} \sum_{l=0}^{n-j} n \quad n-j \quad(-1)^{l}\left(\frac{B_{G\left(y_{k} ; \alpha, \beta, \theta\right)}(a, b)}{B(a, b)}\right)^{j+l} \\
& =\sum_{j=k}^{n} \sum_{l=0}^{n-j} n{ }_{j} n-j \frac{(-1)^{l}}{[B(a, b)]^{j+l}}\left(B_{G\left(y_{k} ; \alpha, \beta, \theta\right)}(a, b)\right)^{j+l} .
\end{aligned}
$$

\subsection{Rényi Entropy}

Rényi entropy (1960) is an extension of Shannon entropy. Rényi entropy is defined to be $H_{v}\left(f_{\text {BLFRG }}(x ; \alpha, \beta, \theta, a, b)\right)=\frac{\log \left(\int_{0}^{\infty} f_{B L F R G}^{v}(x ; \alpha, \beta, \theta, a, b) d x\right)}{1-v}$, where $v>0$, and $v \neq 1$.

Rényi entropy tends to Shannon entropy as $v \rightarrow 1$. Note that by using the series representations in equations (12) and (15), we have

$$
\begin{aligned}
f_{\text {BLFRG }}^{v}(x) & =\frac{(1-\theta)^{b v}}{B^{v}(a, b)} \sum_{j, k=0}^{\infty} \sum_{r=0}^{v} \frac{(-1)^{k} \Gamma(v(a+b)+j)}{j ! \Gamma(v(a+b))} \\
& \times\left(\begin{array}{c}
v(a-1) \\
k
\end{array}\right)\left(\begin{array}{l}
v \\
r
\end{array}\right) \theta^{j} a^{v-r} b^{r} x^{r} e^{-(k+j+b v)\left(\alpha x+\frac{\beta}{2} x^{2}\right)} \\
& =\frac{(1-\theta)^{b v}}{B^{v}(a, b)} \sum_{j, k, s=0}^{\infty} \sum_{r=0}^{v} \frac{(-1)^{k+r+s} \Gamma(v(a+b)+j)\left[(k+j+b v)\left(\frac{\beta}{2}\right)\right]^{s}}{j ! s ! \Gamma(v(a+b))} \\
& \times\left(\begin{array}{c}
v(a-1) \\
k
\end{array}\right)\left(\begin{array}{l}
v \\
r
\end{array}\right) \theta^{j} a^{v-r} b^{r} x^{r+2 s} e^{-(k+j+b v)\left(\alpha x+\frac{\beta}{2} x^{2}\right)} .
\end{aligned}
$$

Now, 


$$
\begin{aligned}
\int_{0}^{\infty} f_{B L F R G}^{v}(x) d x & =\frac{(1-\theta)^{b v}}{B^{v}(a, b)} \sum_{j, k, s=0}^{\infty} \sum_{r=0}^{v} \frac{(-1)^{k+r+s} \Gamma(v(a+b)+j)\left[(k+j+b v)\left(\frac{\beta}{2}\right)\right]^{s}}{j ! s ! \Gamma(v(a+b))} \\
& \times\left(\begin{array}{c}
v(a-1) \\
k
\end{array}\right)\left(\begin{array}{l}
v \\
r
\end{array}\right) \theta^{j} \frac{a^{v-r} b^{r} \Gamma(r+2 s+1)}{[\alpha(k+j+b v)]^{r+2 s+1}} .
\end{aligned}
$$

Consequently, Rényi entropy for the BLFRG distribution is

$$
\begin{aligned}
H_{v}\left(f_{B L F R G}(x)\right)=\frac{1}{1-v} \log \left[\frac{(1-\theta)^{b v}}{B^{v}(a, b)} \sum_{j, k, s=0}^{\infty} \sum_{r=0}^{v} \frac{(-1)^{k+r+s} \Gamma(v(a+b)+j) a^{v-r} b^{r}}{j ! s ! \Gamma(v(a+b))}\right. \\
\left.\times\left(\begin{array}{c}
v(a-1) \\
k
\end{array}\right)\left(\begin{array}{c}
v \\
r
\end{array}\right) \frac{\theta^{j}[(k+j+b v)(\beta / 2)]^{s}}{[\alpha(k+j+b v)]^{r+2 s+1}} \Gamma(r+2 s+1)\right],
\end{aligned}
$$

for $v \neq 1, v>0$.

\section{$5.3 s$-Entropy}

The $s$-entropy for BLFRG distribution is defined by

$H_{s}\left(f_{B L F R G}(x ; \alpha, \beta, \theta, a, b)\right)=\left\{\begin{array}{cc}\frac{1}{s-1}\left[1-\int_{0}^{\infty} f_{B L F R G}^{s}(x ; \alpha, \beta, \theta, a, b) d x\right] & \text { if } \mathrm{s} \neq 1, \mathrm{~s}>0, \\ E\left[-\log f_{B L F R G}(X)\right] & \text { if } \mathrm{s}=1,\end{array}\right.$ where $\int_{0}^{\infty} f_{B L F R G}^{s}(x ; \alpha, \beta, \theta, a, b) d x$ is given below:

$$
\begin{aligned}
\int_{0}^{\infty} f_{\text {BLFRG }}^{s}(x) d x & =\frac{(1-\theta)^{b s}}{B^{s}(a, b)} \sum_{j, k, p=0}^{\infty} \sum_{r=0}^{s} \frac{(-1)^{k+r+p} \Gamma(s(a+b)+j)\left[(k+j+b s)\left(\frac{\beta}{2}\right)\right]^{p}}{j ! p ! \Gamma(s(a+b))} \\
& \times\left(\begin{array}{c}
s(a-1) \\
k
\end{array}\right)\left(\begin{array}{l}
s \\
r
\end{array}\right) \theta^{j} \frac{a^{s-r} b^{r} \Gamma(r+2 p+1)}{[\alpha(k+j+b s)]^{r+2 p+1}} .
\end{aligned}
$$

\section{Estimation and Inference}

In this section, we present the maximum likelihood estimates (MLEs) of the parameters of the BLFRG distribution from complete samples only. Let $x_{1}, x_{2}, \ldots, x_{n}$ be a random sample of size $n$ from $\operatorname{BLFRG}(x ; \varphi)$, distribution where $\varphi=(\alpha, \beta, \theta, a, b)^{T}$ is the vector of model parameters. The $\log$-likelihood function $\ell_{n}=\log L$ for the vector of parameters $\varphi=(\alpha, \beta, \theta, a, b)$ can be written as 


$$
\begin{aligned}
\ell=\log L & =n b \log (1-\theta)+\sum_{i=1}^{n} \log \left(\alpha+\beta x_{i}\right)-n \log \Gamma(a)-n \log \Gamma(b)+n \log \Gamma(a+b) \\
& -b \alpha \sum_{i=1}^{n} x_{i}-\frac{b \beta}{2} \sum_{i=1}^{n} x_{i}^{2}+(a-1) \sum_{i=1}^{n} \log \left(1-e^{t_{i}}\right) \\
& -(a+b) \sum_{i=1}^{n} \log \left(1-\theta e^{t_{i}}\right),
\end{aligned}
$$

where $t_{i}=-\left(\alpha x_{i}+\frac{\beta}{2} x_{i}^{2}\right)$. The associated score function is given by

$$
U_{n}(\varphi)=\left(\frac{\partial \ell}{\partial \alpha}, \frac{\partial \ell}{\partial \beta}, \frac{\partial \ell}{\partial \theta}, \frac{\partial \ell}{\partial a}, \frac{\partial \ell}{\partial b}\right)^{T} .
$$

The log-likelihood can be maximized either directly or by solving the nonlinear likelihood equations obtained by differentiating the log likelihood function. The components of the score vector are given by

$$
\begin{aligned}
\frac{\partial \log \ell}{\partial \alpha}= & \sum_{i=1}^{n} \frac{1}{\left(\alpha+\beta x_{i}\right)}-b \sum_{i=1}^{n} x_{i}+(a-1) \sum_{i=1}^{n} \frac{x_{i} e^{t_{i}}}{\left(1-e^{t_{i}}\right)} \\
& -(a+b) \sum_{i=1}^{n} \frac{\theta x_{i} e^{t_{i}}}{\left(1-\theta e^{t_{i}}\right)}, \\
\frac{\partial \ell}{\partial \beta}= & \sum_{i=1}^{n} \frac{x_{i}}{\left(\alpha+\beta x_{i}\right)}-\frac{b}{2} \sum_{i=1}^{n} x_{i}^{2}+\frac{(a-1)}{2} \sum_{i=1}^{n} \frac{x_{i}^{2} e^{t_{i}}}{\left(1-e^{t_{i}}\right)}-\frac{(a+b)}{2} \sum_{i=1}^{n} \frac{\theta x_{i}^{2} e^{t_{i}}}{\left(1-\theta e^{t_{i}}\right)}, \\
\frac{\partial \ell}{\partial \theta}= & \frac{-n b}{1-\theta}+(a+b) \sum_{i=1}^{n} \frac{e^{t_{i}}}{\left(1-\theta e^{t_{i}}\right)}, \\
\frac{\partial \ell}{\partial a}= & \sum_{i=1}^{n} \log \left(1-e^{t_{i}}\right)-n \psi(a)+n \psi(a+b)-\sum_{i=1}^{n} \log \left(1-\theta e^{t_{i}}\right),
\end{aligned}
$$

and

$$
\frac{\partial \ell}{\partial b}=n \log (1-\theta)-\alpha \sum_{i=1}^{n} x_{i}-\frac{\beta}{2} \sum_{i=1}^{n} x_{i}^{2}-n \psi(b)+n \psi(a+b)-\sum_{i=1}^{n} \log \left(1-\theta e^{t_{i}}\right),
$$

respectively. The maximum likelihood estimate (MLE) of $\varphi$, say $\widehat{\varphi}$ is obtained by solving the nonlinear system $U_{n}(\varphi)=0$. These equations cannot be solved analytically, and statistical 
software can be used to solve them numerically via iterative methods. We can use iterative techniques such as a Newton-Raphson type algorithm to obtain the estimate $\widehat{\varphi}$.

The convergence of the estimation procedures often depends on the choice of starting or initial values of the parameters, so one has to be cautious or care must be taken when obtaining the numerical approximations of the expected information matrix. For methods such as the BFGS, approximation of the Hessian matrix is used for the computations of each iteration and this approximation may not be reliable when convergence of the methods occurs too fast, thereby leading to an unreliable approximate Hessian matrix. Other methods of estimation such as generalized method of moments to obtain initial values followed by Newton or quasiNewton methods to obtain better and reliable parameter estimates may be used. In this paper, we maximize the likelihood function using NLmixed in SAS and nlm in R. The function was applied and executed for wide range of initial values. This process often results or lead to more than one maximum, however, in these cases, we take the MLEs corresponding to the largest value of the maxima. In a few cases, no maximum was identified for the selected initial values. In these cases, a new initial value was tried in order to obtain a maximum.

For interval estimation and hypothesis tests on the model parameters, we require the information matrix. The Fisher information matrix is given by

$$
\mathbf{J}(\varphi)=\left[\mathbf{J}_{\theta_{i}, \theta_{j}}\right]_{5 X 5}=E\left(-\frac{\partial^{2} \log L}{\partial \theta_{i} \partial \theta_{j}}\right), i, j=1,2,3,4,5 \text {, can be numerically obtained. The total }
$$

Fisher information matrix $n \mathbf{J}(\varphi)$ can be approximated by

$$
\mathbf{I}_{n}(\hat{\varphi}) \approx\left[-\left.\frac{\partial^{2} \ell_{n}}{\partial \theta_{i} \partial \theta_{j}}\right|_{\varphi=\hat{\varphi}}\right]_{5 X 5}, \quad i, j=1,2,3,4,5 \text {. }
$$

For a given set of observations, the matrix given in the equation above is obtained after the convergence of the Newton-Raphson procedure. Applying the usual large sample approximation, MLE of $\varphi$, that is $\widehat{\varphi}$ can be treated as being approximately $N_{5}\left(\varphi, J_{n}(\varphi)^{-1}\right)$, where $J_{n}(\varphi)=E\left[I_{n}(\varphi)\right]$. Under conditions that are fulfilled for parameters in the interior of the parameter space but not on the boundary, the asymptotic distribution of $\sqrt{\mathrm{n}}(\widehat{\varphi}-\varphi)$ is $N_{5}\left(0, J(\varphi)^{-1}\right)$, where $J(\varphi)=\lim _{n \rightarrow \infty} n^{-1} I_{n}(\varphi)$ is the unit information matrix. This asymptotic behavior remains valid if $J(\varphi)$ is replaced by the average sample information matrix evaluated at $\hat{\varphi}$, say $\mathrm{n}^{-1} \mathrm{I}_{\mathrm{n}}(\widehat{\varphi})$. The estimated asymptotic multivariate normal $\mathrm{N}_{5}\left(\varphi, I_{n}(\hat{\varphi})^{-1}\right)$ distribution of $\hat{\varphi}$ can be used to construct approximate confidence intervals for the parameters and for the hazard rate and survival functions. An $100(1-\xi) \%$ asymptotic confidence interval for each parameter $\varphi_{r}$ is given by

$$
A C I_{r}=\left(\hat{\varphi}_{r}-z_{\frac{\xi}{2}} \sqrt{\hat{I}_{r r}}, \hat{\varphi}_{r}+z_{\frac{\xi}{2}} \sqrt{\hat{I}_{r r}}\right)
$$


where $\hat{I}_{r r}$ is the $(r, r)$ diagonal element of $I_{n}(\hat{\varphi})^{-1}$ for $r=1,2,3,4,5$, and $\mathrm{z}_{\frac{\zeta}{2}}$ is the $1-\frac{\zeta}{2}$ quantile of the standard normal distribution.

We can use the likelihood ratio (LR) test to compare the fit of the BLFRG distribution with its sub-models for a given data set. For example, to test $a=b=1$, the LR statistic is $\omega=2[\ln (L(\hat{\alpha}, \hat{\beta}, \hat{\theta}, \hat{a}, \hat{b}))-\ln (L(\tilde{\alpha}, \widetilde{\beta}, \tilde{\theta}, 1,1))]$, where $\hat{\alpha}, \quad \hat{\beta}, \quad \hat{\theta}, \quad \hat{a}$ and $\hat{b}, \quad$ are the unrestricted estimates, and $\tilde{\alpha}, \tilde{\beta}$, and $\tilde{\theta}$ are the restricted estimates. The LR test rejects the null hypothesis if $\omega>\chi_{\varepsilon}^{2}$, where $\chi_{\varepsilon}^{2}$ denote the upper $100 \varepsilon \%$ point of the $\chi^{2}$ distribution with 2 degrees of freedom.

\section{Simulation}

In this section, we examine the performance of the BLFRG distribution by conducting various simulations for different sample sizes. We simulate 1000 samples for the true parameters values $I: \alpha=4, \beta=3, \theta=0.6, a=6, b=2 \quad$ and II : $\alpha=2, \beta=5, \theta=0.8, a=7, b=3$. Table 3 lists the mean MLEs of the five model parameters along with their respective root mean squared errors (RMSE) and Bias for sample sizes $n=50, n=100, n=200, n=400$ and $n=800$. For a parameter $\varphi$ and its estimate $\hat{\varphi}$. The root-mean-square error (RMSE) of the MLE $\hat{\varphi}$ is given by

$$
R M S E=\sqrt{\frac{\sum_{i=1}^{N}\left(\hat{\varphi}_{i}-\varphi\right)^{2}}{N} .}
$$

The average bias of the MLE $\hat{\varphi}$ of the parameter $\varphi=\alpha, \beta, \theta, a, b$ is given by

$$
\operatorname{Bias}(\hat{\varphi})=\frac{\sum_{i=1}^{N}\left(\hat{\varphi}_{i}-\varphi\right)}{N} .
$$

From the results in Table 3, we can verify that as the sample size $n$ increases, the RMSEs decay toward zero. We also observe that for all the parametric values, the biases decrease as the sample size $n$ increases.

Table 3 Monte Carlo Simulation Results: Mean Estimates, Biases and RMSEs

\begin{tabular}{cccccccc}
\hline & \multicolumn{3}{c}{ I } & \multicolumn{3}{c}{ II } \\
\hline & $\mathrm{n}$ & Mean & RMSE & Bias & Mean & RMSE & Bias \\
\hline$\alpha$ & 50 & 4.5154501 & 11.2114814 & 1.51545007 & 2.7610646 & 5.6103511 & -2.238935364 \\
& 100 & 4.0134972 & 5.8391564 & 3.41349715 & 2.7879148 & 3.7391658 & 1.987914814 \\
& 200 & 4.2204255 & 5.160537 & -1.77957451 & 3.005777 & 3.3657174 & -3.994223016 \\
& 400 & 4.2528155 & 3.202751 & 2.25281551 & 2.7401263 & 2.5887505 & -0.259873698
\end{tabular}




\begin{tabular}{cccccccc}
\multicolumn{7}{l}{336} & \multicolumn{7}{c}{ Beta Linear Failure Rate Geometric Distribution } \\
\hline & 800 & 4.2572026 & 2.4847826 & 0.25720265 & 2.4998983 & 2.164769 & 0.499898342 \\
\hline$\beta$ & 50 & 24.4932256 & 77.2488562 & 23.89322562 & 21.3369212 & 91.5279917 & 20.536921179 \\
& 100 & 22.0585346 & 55.7548951 & 16.05853461 & 16.5111159 & 38.0552367 & 9.511115884 \\
& 200 & 15.7179659 & 38.2994578 & 13.71796591 & 11.7575803 & 20.1213485 & 8.75758031 \\
& 400 & 11.1254503 & 23.2524148 & 7.12545026 & 8.8636964 & 12.1680447 & 6.863696406 \\
& 800 & 7.1381057 & 12.8558492 & 4.13810566 & 7.0963682 & 9.034725 & 2.096368177 \\
\hline$\theta$ & 50 & 0.4105315 & 0.4434283 & -5.58946855 & 0.485253 & 0.5279769 & -6.514747045 \\
& 100 & 0.5322302 & 0.3896812 & -1.46776981 & 0.6362691 & 0.4049937 & -2.363730908 \\
& 200 & 0.5726993 & 0.3419892 & -3.42730067 & 0.7135356 & 0.2956052 & -1.286464446 \\
& 400 & 0.5650341 & 0.3155987 & -2.43496586 & 0.7731983 & 0.1791921 & -4.226801687 \\
& 800 & 0.5789249 & 0.2530794 & -0.02107514 & 0.7908513 & 0.1345934 & -0.009148678 \\
\hline $\mathrm{a}$ & 50 & 12.561048 & 47.8085994 & 10.56104802 & 18.0967032 & 87.1831627 & 15.096703187 \\
& 100 & 13.0250482 & 45.7676797 & 9.02504818 & 12.4131211 & 26.3085505 & 10.413121071 \\
& 200 & 9.56369 & 15.7450963 & 6.56368997 & 10.8098116 & 20.0088847 & 5.809811612 \\
& 400 & 8.1268523 & 10.1831008 & 7.52685231 & 9.0755166 & 12.8955991 & 8.275516625 \\
& 800 & 7.0013257 & 5.4794791 & 1.00132565 & 7.8403358 & 6.3718872 & 0.840335804 \\
\hline $\mathrm{b}$ & 50 & 4.4113233 & 4.9417386 & 0.41132333 & 4.8811163 & 4.7076651 & 2.881116283 \\
& 100 & 2.7784406 & 2.7725411 & -0.22155937 & 3.1912916 & 2.5265626 & -1.808708385 \\
& 200 & 2.2536851 & 1.937578 & 1.65368507 & 2.655721 & 1.4303754 & 1.855720975 \\
& 400 & 2.1096664 & 1.5679672 & -3.89033357 & 2.6224246 & 1.1062168 & -4.377575431 \\
& 800 & 2.1068646 & 1.3347534 & 0.10686455 & 2.7063814 & 0.837099 & -0.293618556 \\
\hline
\end{tabular}

\section{Applications}

In this section, we present examples to illustrate the flexibility of the BLFRG distribution and its sub-models for data modeling. We also compare the five parameters BLFRG distribution to the beta-Weibull-geometric (BWG) distribution (Bidram et al. (2013)). The cdf and pdf of BWG distribution are given by

$$
F(x ; \alpha, \beta, p, a, b)=\frac{1}{B(a, b)} \int_{0}^{1-p e^{-(\beta x)^{\alpha}}} \omega^{a-1}(1-\omega)^{b-1} d \omega,
$$

and

$$
f(x ; \alpha, \beta, p, a, b)=\frac{(1-p)^{b} \alpha \beta^{\alpha} x^{\alpha-1} e^{-b(\beta x)^{\alpha}}\left(1-e^{-(\beta x)^{\alpha}}\right)^{a-1}}{B(a, b)\left(1-p e^{-(\beta x)^{\alpha}}\right)^{a+b}},
$$

respectively, where $x>0, a, b, \alpha, \beta>0$ and $p \in(0,1)$.

The maximum likelihood estimates (MLEs) of the BLFRG parameters $\alpha, \beta, \theta, a$, and $b$ are computed by maximizing the objective function via the subroutine NLMIXED in SAS. The estimated values of the parameters (standard error in parenthesis), -2log-likelihood statistic, 
Akaike Information Criterion, $A I C=2 p-2 \ln (L)$, Bayesian Information Criterion, $B I C=p \ln (n)-2 \ln (L) \quad, \quad$ and Consistent Akaike Information Criterion, $A I C C=A I C+2 \frac{p(p+1)}{n-p-1}$, where $L=L(\hat{\varphi})$ is the value of the likelihood function evaluated at the parameter estimates, $n$ is the number of observations, and $p$ is the number of estimated parameters are presented in Tables 4 and 5, respectively. Also, presented are values of the the goodness-of-fit statistics $W^{*}$ and $A^{*}$, described by Chen and Balakrishnan (1995). These statistics can be used to verify which distribution fits better to the data. In general, the smaller the values of $W^{*}$ and $A^{*}$, the better the fit. Let $G(x ; \Delta)$ be the cdf, where the form of $G$ is known but the k-dimensional parameter vector, say $\Delta$ is unknown. We can obtain the statistics $W^{*}$ and $A^{*}$ as follows:

i. Compute $u_{i}=G\left(x_{i} ; \hat{\Delta}\right)$, where the $x_{i}$ 's are in ascending order;

ii. Compute $y_{i}=\Phi^{-1}\left(u_{i}\right)$, where $\Phi($.$) is the standard normal cdf and \Phi^{-1}($.) its inverse;

iii. Compute $v_{i}=\Phi\left(\left(y_{i}-\bar{y}\right) / s_{y}\right)$, where $\bar{y}=n^{-1} \sum_{i=1}^{n} y_{i}$ and $s_{y}^{2}=(n-1)^{-1} \sum_{i=1}^{n}\left(y_{i}-\bar{y}\right)^{2}$;

iv. Calculate $W^{2}=\sum_{i=1}^{n}\left\{v_{i}-(2 i-1) /(2 n)\right\}^{2}+1 /(12 n)$ and

$$
A^{2}=-n-n^{-1} \sum_{i=1}^{n}\left\{(2 i-1) \log \left(v_{i}\right)+(2 n+1-2 i) \log \left(1-v_{i}\right)\right\}
$$

v. Modify $W^{2}$ into $W^{*}=W^{2}(1+0.5 / n)$ and $A^{2}$ into $A^{*}=A^{2}\left(1+0.75 / n+2.25 / n^{2}\right)$. The sum of squares $S S=\sum_{j=1}^{n}\left[G_{B L F R G}\left(x_{(j)} ; \hat{\alpha}, \hat{\beta}, \hat{\theta}, \hat{a}, \hat{b}\right)-\left(\frac{j-0.375}{n+0.25}\right)\right]^{2}$ that are obtained from the probability plots are also given in the tables. These statistics are used to compare the distributions presented in Tables 4 and 5. Plots of the fitted densities, the histogram and probability plots (Chambers et al. (1983)) of the data are given in Figures 3 and 4. For the probability plot, we plotted $G_{B L F R G}\left(x_{(j)} ; \hat{\alpha}, \hat{\beta}, \hat{\theta}, \hat{a}, \hat{b}\right)$ against $\frac{j-0.375}{n+0.25}, j=1,2, \cdots, n$, where $x_{(j)}$ are the ordered values of the observed data.

\subsection{Time to failure of kevlar 49/epoxy strands tested at various stress level}

The data consists of a real life example is taken from Cooray and Ananda (2008), where 101 data points represent the stress-rupture life of kevlar 49/epoxy strands which are subjected 
to constant sustained pressure at the $90 \%$ stress level until all have failed, so that the complete data set with the exact times of failure is recorded. These failure times in hours, are originally given in Andrews and Herzberg (1985) and Barlow et al. (1984). Initial value for BLFRG model in the $\mathrm{R}$ code are $\alpha=0.1, \beta=1, \theta=0.5, a=0.1, b=0.2$. Estimates of the parameters of BLFRG distribution and its related sub-models (standard error in parentheses), AIC, AICC, BIC, $W^{*}, A^{*}$ and SS for stress-rupture life of kevlar 49/epoxy strands data are given in Table 4.

Table 4 Estimates of Models for Failure Times of Kevlar Data Set

\begin{tabular}{|c|c|c|c|c|c|c|c|c|c|c|c|c|}
\hline \multirow[b]{2}{*}{ Model } & \multicolumn{5}{|c|}{ Estimates } & \multicolumn{7}{|c|}{ Statistics } \\
\hline & $\alpha$ & $\beta$ & $\theta$ & $a$ & $\mathrm{~b}$ & $-2 \log L$ & AIC & AICC & $\mathrm{BIC}$ & $\mathrm{W}^{*}$ & $A^{*}$ & SS \\
\hline BLFRG & $\begin{array}{c}0.0277 \\
(0.0669)\end{array}$ & $\begin{array}{c}0.0096 \\
(0.0300)\end{array}$ & $\begin{array}{c}0.8602 \\
(0.1805)\end{array}$ & $\begin{array}{c}0.8920 \\
(0.1505)\end{array}$ & $\begin{array}{c}4.0777 \\
(2.5645)\end{array}$ & 205.05 & 215.05 & 215.69 & 228.13 & 0.1586 & 0.9191 & 0.1478 \\
\hline ELFRG & $\begin{array}{c}0.0382 \\
(0.0482)\end{array}$ & $\begin{array}{c}0.1166 \\
(0.0766)\end{array}$ & $\begin{array}{c}0.8950 \\
(0.0881)\end{array}$ & $\begin{array}{c}0.6677 \\
(0.1649)\end{array}$ & $\begin{array}{l}1 \\
-\end{array}$ & 206.01 & 214.01 & 214.64 & 224.47 & 0.1627 & 0.9598 & 0.1429 \\
\hline LFRG & $\begin{array}{c}0.1193 \\
(0.1379)\end{array}$ & $\begin{array}{c}0.1240 \\
(0.0678)\end{array}$ & $\begin{array}{c}0.8839 \\
(0.1205)\end{array}$ & $\begin{array}{l}1 \\
-\end{array}$ & $\begin{array}{l}1 \\
-\end{array}$ & 208.87 & 214.87 & 215.50 & 222.71 & 0.2481 & 1.3704 & 0.2070 \\
\hline EG & $\begin{array}{c}0.9305 \\
(0.2007)\end{array}$ & $\begin{array}{l}0 \\
-\end{array}$ & $\begin{array}{c}0.0896 \\
(0.3366)\end{array}$ & $\begin{array}{l}1 \\
-\end{array}$ & $\begin{array}{l}1 \\
-\end{array}$ & 206.89 & 210.89 & 211.52 & 216.12 & 0.1936 & 1.0894 & 0.1753 \\
\hline $\mathrm{E}$ & $\begin{array}{c}0.9758 \\
(0.0971)\end{array}$ & $\begin{array}{l}0 \\
-\end{array}$ & $\begin{array}{l}0 \\
-\end{array}$ & $\begin{array}{l}1 \\
-\end{array}$ & $\begin{array}{l}1 \\
-\end{array}$ & 206.96 & 208.96 & 209.59 & 211.57 & 0.1809 & 1.0283 & 0.1645 \\
\hline $\mathrm{BR}$ & $\begin{array}{l}0 \\
-\end{array}$ & $\begin{array}{c}0.3484 \\
(0.1908)\end{array}$ & $\begin{array}{l}0 \\
-\end{array}$ & $\begin{array}{c}0.3127 \\
(0.0356)\end{array}$ & $\begin{array}{c}1.0000 \\
(0.4410)\end{array}$ & 215.39 & 221.39 & 222.03 & 229.24 & 0.1389 & 1.0130 & 0.2187 \\
\hline $\mathrm{R}$ & $\begin{array}{l}0 \\
-\end{array}$ & $\begin{array}{c}0.8730 \\
(0.0869)\end{array}$ & $\begin{array}{l}0 \\
-\end{array}$ & $\begin{array}{l}1 \\
-\end{array}$ & $\begin{array}{l}1 \\
-\end{array}$ & 360.46 & 362.46 & 363.09 & 365.07 & 0.1206 & 0.9184 & 3.5326 \\
\hline BWG & $\begin{array}{c}\alpha \\
1.2114 \\
(0.3379)\end{array}$ & $\begin{array}{c}\beta \\
0.0185 \\
(0.0826)\end{array}$ & $\begin{array}{c}\mathrm{p} \\
0.6935 \\
(1.7747)\end{array}$ & $\begin{array}{c}\mathrm{a} \\
0.6705 \\
(0.2723)\end{array}$ & $\begin{array}{c}b \\
23.2461 \\
(0.0300)\end{array}$ & 205.24 & 215.24 & 215.88 & 228.32 & 0.1515 & 0.8949 & 0.1505 \\
\hline
\end{tabular}

The asymptotic covariance matrix of the MLEs of the BLFRG model parameters, which is the inverse of the observed Fisher information matrix $\mathbf{I}_{n}^{-1}(\varphi)$ is given by:

$$
\left(\begin{array}{ccccc}
0.004469555 & 0.0022450617 & -0.013057828 & -0.003277698 & -0.31659795 \\
0.002245062 & 0.0009007749 & -0.008667904 & -0.001059272 & -0.08870874 \\
-0.013057828 & -0.0086679039 & 0.032578647 & 0.026711818 & 1.29910271 \\
-0.003277698 & -0.001059272 & 0.026711818 & 0.022655984 & -0.13344524 \\
-0.316597948 & -0.0887087408 & 1.29910271 & -0.13344524 & 6.57665597
\end{array}\right),
$$


and the $95 \%$ asymptotic confidence intervals for the model parameters are given by $\alpha \in(0.0277 \pm 1.96 \times 0.0669), \beta \in(0.0096 \pm 1.96 \times 0.0300), \theta \in(0.8602 \pm 1.96 \times 0.1805)$, $a \in(0.8920 \pm 1.96 \times 0.1505)$, and $b \in(4.0777 \pm 1.96 \times 2.5645)$, respectively.

Plots of the fitted densities and the histogram, observed probability vs predicted probability, and empirical survival function for the data are given in Figure 3.
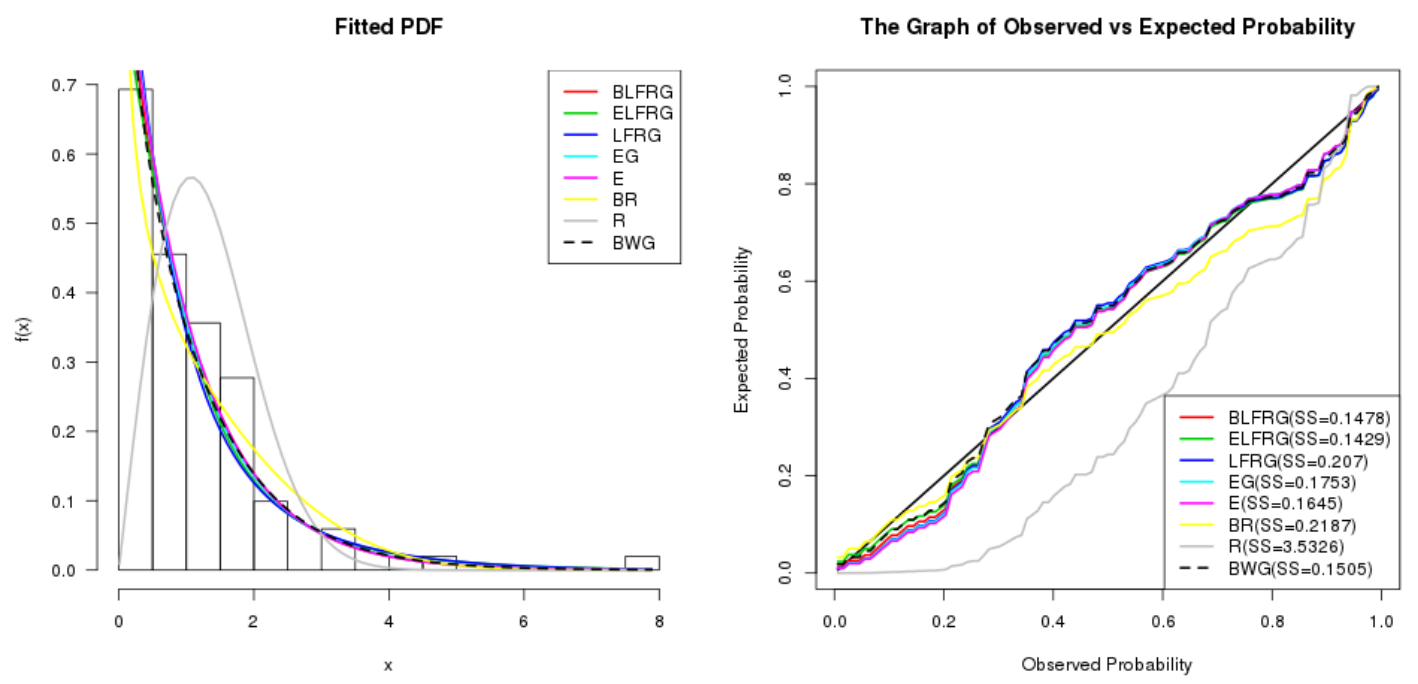

Figure 3: Fitted Densities and Probability Plots

The LR test statistic of the hypotheses $H_{0}$ : BR against $H_{a}$ : BLFRG and $H_{0}$ : LFRG against $H_{a}$ : BLFRG are $10.3(\mathrm{p}$-value $=0.0056)$ and $3.8(\mathrm{p}$-value $=0.1485)$. We can conclude that there is a significant difference between BLFRG and BR distributions. Considering the values of the statistics AIC, BIC and the values of SS given in Table 4, we observe that the BLFRG distribution gives a better fit for the data. The values of the goodnessof-fit statistics $W^{*}$ and $A^{*}$ shows that the BLFRG distribution is a better fit that its submodels except for the staistics $W^{*}$ for the BR and $\mathrm{R}$ distributions. When the BLFRG distribution is compared to the non-nested five parameter BWG distribution, it is seen that the BLFRG distribution is a competitive distribution.

\subsection{Fatigue fracture of kevlar 373/epoxy}

The data set is on the life of fatigue fracture of Kevlar 373/epoxy that are subject to constant pressure at the $90 \%$ stress level until all had failed. The complete data with the exact times of failure was also studied by Andrews and Herzberg (1985), and Barlow, Toland and Freeman (1984). Initial value for BLFRG model in the $\mathrm{R}$ code are $\alpha=1, \beta=1, \theta=0.3, a=4, b=2$. Estimates of the parameters of BLFRG distribution and its 
related sub-models (standard error in parentheses), AIC, AICC, BIC, $W^{*}, A^{*}$ and SS for the fatigue fracture of Kevlar 373/epoxy data are given in Table 5.

Table 5 Estimates of Models for Failure Times of Kevlar Data Set

\begin{tabular}{|c|c|c|c|c|c|c|c|c|c|c|c|c|}
\hline \multirow[b]{2}{*}{ Model } & \multicolumn{5}{|c|}{ Estimates } & \multicolumn{7}{|c|}{ Statistics } \\
\hline & $\alpha$ & $\beta$ & $\theta$ & $\mathrm{a}$ & $\mathrm{b}$ & $-2 \log L$ & AIC & AICC & BIC & $\mathrm{W}^{*}$ & $\mathrm{~A}^{*}$ & SS \\
\hline BLFRG & $\begin{array}{c}0.0057 \\
(0.0142)\end{array}$ & $\begin{array}{c}0.0310 \\
(0.0656)\end{array}$ & $\begin{array}{c}0.9013 \\
(0.1654)\end{array}$ & $\begin{array}{c}0.9607 \\
(0.3277)\end{array}$ & $\begin{array}{c}1.6459 \\
(0.9600)\end{array}$ & 241.10 & 251.10 & 251.95 & 262.75 & 0.0723 & 0.4207 & 0.0672 \\
\hline ELFRG & $\begin{array}{c}0.0134 \\
(0.0218)\end{array}$ & $\begin{array}{c}0.0909 \\
(0.0464)\end{array}$ & $\begin{array}{c}0.8557 \\
(0.1033)\end{array}$ & $\begin{array}{c}0.9919 \\
(0.3286)\end{array}$ & $\begin{array}{l}1 \\
-\end{array}$ & 242.12 & 250.12 & 250.98 & 259.44 & 0.0891 & 0.5141 & 0.0900 \\
\hline LFRG & $\begin{array}{c}0.0138 \\
(0.0162)\end{array}$ & $\begin{array}{c}0.0909 \\
(0.0462)\end{array}$ & $\begin{array}{c}0.8566 \\
(0.0935)\end{array}$ & $\begin{array}{l}1 \\
-\end{array}$ & $\begin{array}{l}1 \\
-\end{array}$ & 242.12 & 248.12 & 248.98 & 255.11 & 0.0892 & 0.5149 & 0.0896 \\
\hline GLFR & & & $\begin{array}{l}0 \\
-\end{array}$ & $\begin{array}{c}1 \\
(0.4031)\end{array}$ & $\begin{array}{l}1 \\
-\end{array}$ & 248.97 & 254.97 & 255.83 & 261.96 & 0.1818 & 1.0528 & 0.2161 \\
\hline LFR & & & $\begin{array}{l}0 \\
-\end{array}$ & $\begin{array}{l}1 \\
-\end{array}$ & $\begin{array}{l}1 \\
-\end{array}$ & 248.97 & 252.97 & 253.83 & 257.63 & 0.1818 & 1.0528 & 0.2161 \\
\hline $\mathrm{E}$ & $\begin{array}{c}0.5104 \\
(0.0585)\end{array}$ & $\begin{array}{l}0 \\
-\end{array}$ & $\begin{array}{l}0 \\
-\end{array}$ & $\begin{array}{l}1 \\
-\end{array}$ & $\begin{array}{l}1 \\
-\end{array}$ & 254.23 & 256.23 & 257.09 & 258.56 & 0.1193 & 0.7074 & 0.5612 \\
\hline BRG & $\begin{array}{l}0 \\
-\end{array}$ & $\begin{array}{c}0.0241 \\
(0.0799)\end{array}$ & $\begin{array}{c}0.9039 \\
(0.2469)\end{array}$ & & $\begin{array}{r}1.735 \\
(1.336\end{array}$ & 242.25 & 250.25 & 251.11 & 259.57 & 0.0852 & 0.5071 & 0.0775 \\
\hline BR & $\begin{array}{l}0 \\
-\end{array}$ & $\begin{array}{c}0.2004 \\
(0.1471)\end{array}$ & $\begin{array}{l}0 \\
-\end{array}$ & & $\begin{array}{c}1.0000 \\
(0.6425)\end{array}$ & 251.00 & 257.00 & 257.86 & 264.00 & 0.2221 & 1.2748 & 0.2768 \\
\hline $\mathrm{BE}$ & $\begin{array}{c}0.7028 \\
(0.8893)\end{array}$ & $\begin{array}{l}0 \\
-\end{array}$ & $\begin{array}{l}0 \\
-\end{array}$ & $\begin{array}{c}1.7095 \\
(0.3083)\end{array}$ & $\begin{array}{c}1.0000 \\
(1.4067)\end{array}$ & 244.49 & 250.49 & 251.34 & 257.48 & 0.1167 & 0.6935 & 0.1083 \\
\hline $\mathrm{RG}$ & $\begin{array}{l}0 \\
-\end{array}$ & $\begin{array}{c}0.0809 \\
(0.0464)\end{array}$ & $\begin{array}{c}0.8975 \\
(0.0668)\end{array}$ & $\begin{array}{l}1 \\
-\end{array}$ & $\begin{array}{l}1 \\
-\end{array}$ & 245.27 & 249.27 & 250.13 & 253.94 & 0.1197 & 0.7045 & 0.0690 \\
\hline $\mathrm{R}$ & $\begin{array}{l}0 \\
-\end{array}$ & $\begin{array}{c}0.3183 \\
(0.0365)\end{array}$ & $\begin{array}{l}0 \\
-\end{array}$ & $\begin{array}{l}1 \\
-\end{array}$ & $\begin{array}{l}1 \\
-\end{array}$ & 274.64 & 276.64 & 277.50 & 278.97 & 0.2086 & 1.2016 & 1.1748 \\
\hline BWG & $\begin{array}{c}\alpha \\
1.2253 \\
(0.4651)\end{array}$ & $\begin{array}{c}\beta \\
0.0213 \\
(0.1123) \\
\end{array}$ & $\begin{array}{c}\mathrm{p} \\
0.7658 \\
(1.6878)\end{array}$ & $\begin{array}{c}\mathrm{a} \\
1.2534 \\
(0.7125)\end{array}$ & $\begin{array}{c}\mathrm{b} \\
14.2049 \\
(0.0166)\end{array}$ & 244.03 & 254.03 & 254.89 & 265.69 & 0.1125 & 0.6660 & 0.1001 \\
\hline
\end{tabular}

The asymptotic covariance matrix of the MLEs of the BLFRG model parameters, which is the inverse of the observed Fisher information matrix $\mathbf{I}_{n}^{-1}(\varphi)$ is given by: 


$\left(\begin{array}{ccccc}0.0002030163 & 0.0007122536 & -0.001686961 & 0.002209248 & -0.007815576 \\ 0.0007122536 & 0.0043077296 & -0.010100564 & -0.001132638 & -0.052810386 \\ -0.001686961 & -0.0101005639 & 0.027355324 & 0.010792958 & 0.096876446 \\ 0.002209248 & -0.0011326379 & 0.010792958 & 0.107363466 & 0.001972743 \\ -0.0078155761 & -0.0528103862 & 0.096876446 & 0.001972743 & 0.921594731\end{array}\right)$,

and the $95 \%$ asymptotic confidence intervals for the model parameters are given by $\alpha \in(0.0057 \pm 1.96 \times 0.0142), \quad \beta \in(0.0310 \pm 1.96 \times 0.0656), \theta \in(0.9013 \pm 1.96 \times 0.1654)$, $a \in(0.9607 \pm 1.96 \times 0.3277)$, and $b \in(1.6459 \pm 1.96 \times 0.9600)$, respectively.

Plots of the fitted densities and the histogram, observed probability vs predicted probability, and empirical survival function for the data are given in Figure 4.
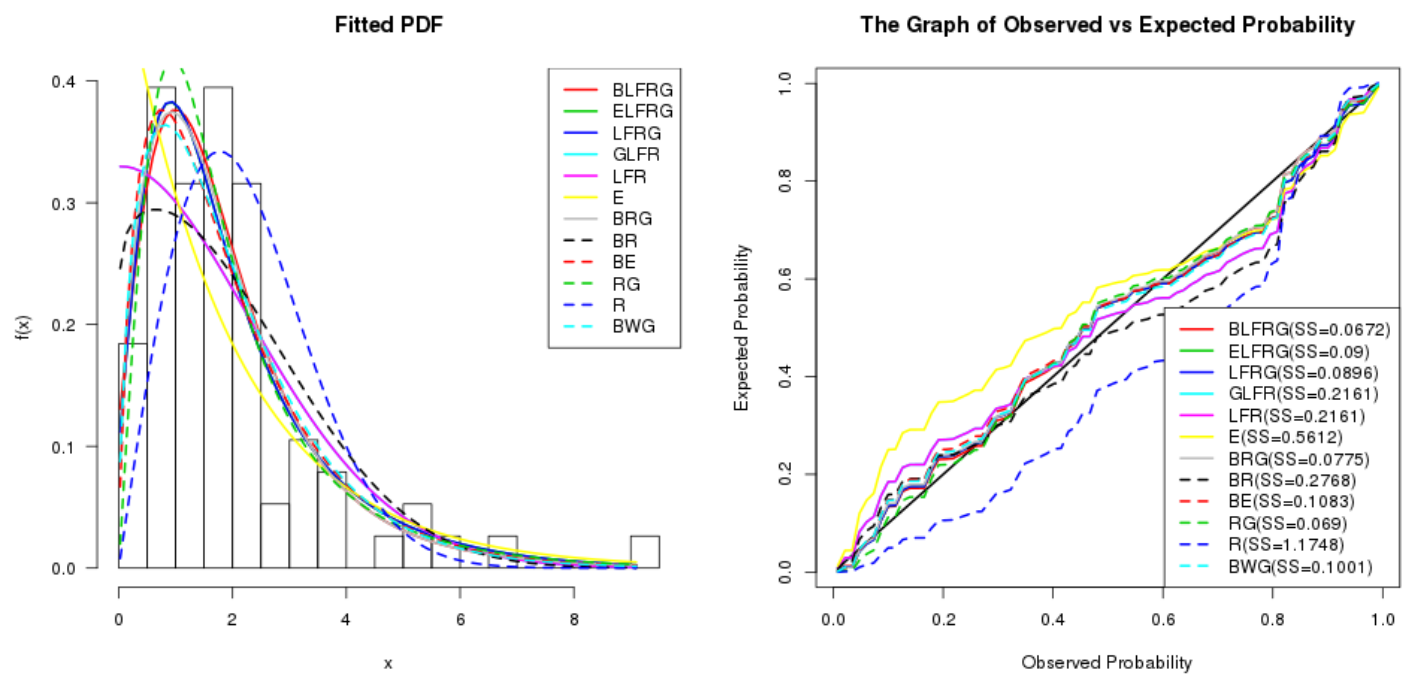

Figure 4: Fitted Densities and Probability Plots

The LR test statistic of the hypotheses $H_{0}:$ BR against $H_{a}:$ BLFRG and $H_{0}:$ LFRG against $H_{a}$ : BLFRG are $9.9(\mathrm{p}$-value $=0.007)$ and $7.9(\mathrm{p}$-value $=0.0486)$. We can conclude that there are significant differences between BLFRG and BR distributions as well as between BLFRG and LFRG distributions. Considering the values of the statistics AIC, BIC, $W^{*}, A^{*}$ and the values of SS given in Table 5, we observe that the BLFRG distribution gives a better fit for the data. When the BLFRG distribution is compared to the non-nested five parameter BWG distribution, it is seen that the BLFRG distribution is a better fit. 


\section{Concluding Remarks}

A new and generalized linear failure rate distribution called the beta linear failure rate geometric (BLFRG) distribution is proposed and studied. The BLFRG distribution has several well known distributions including the LFRG, LFR, EG, RG, Rayleigh and exponential distributions as special cases. The density of this new class of distributions can be expressed as a linear combination of BLFR density functions. The BLFRG distribution possesses hazard function with flexible behavior. We also obtain closed form expressions for the moments, mean and median deviations, distribution of order statistics and entropy. Maximum likelihood estimation technique is used to estimate the model parameters. Finally, the BLFRG distribution is fitted to real data sets to illustrate its applicability and usefulness.

\section{Acknowledgements}

The authors would like to thank the editor and the referees for carefully reading the paper and for their valuable comments, which greatly improved the presentation of this paper.

\section{References}

[1] Adamidis, K., and Loukas, S. (1998). A Lifetime Distribution with Decreasing Failure Rate, Statistics and Probability Letters, 39, 35-42.

[2] Alven, W. H. (1964). Reliability Engineering by ARINC. Prentice Hall, New Jersey.

[3] Andrews, A. D., and Herzberg, A. M. (1985). Data: A Collection of Problems from Many Fields for the Student and Research Worker. Springer Series in Statistics, New York.

[4] Barlow, R. E., Toland, R. H. and Freeman, T. (1984). A Bayesian Analysis of StressRupture Life of Kevlar 49/epoxy Spherical Pressure Vessel. In D. Dwivedi (Ed.), Proceedings of the Canadian Conference in Applied Statistics. Marcel Dekker, New York.

[5] Barreto-Souza, W., Morais, A. L., Cordeiro, G. M. (2011). The Weibull-Geometric Distribution. Journal of Statistical Computation and Simulation, 81, 645-657.

[6] Barreto-Souza, W., Santos A. H. S., Cordeiro, G. M. (2010). The Beta Generalized Exponential Distribution. Journal of Statistical Computation and Simulation, 80, 159-172.

[7] Bidram, H., Behboodian, J., and Towhidi, M. (2013). The Beta Weibull-Geometric Distribution. Journal of Statistical Computation and Simulation, 83(1), 52-67.

[8] Bidram, H. (2012) The Beta Exponential-Geometric Distribution. Communications in Statistics-Simulation and Computation, 41, 1606-1622. 
[9] Bonferroni, C. E. (1930). Elementi di Statistica Generale. Seeber, Firenze.

[10] Chambers, J.M., Cleveland, W.S., Kleiner, B. and Tukey, P.A. (1983). Graphical Methods for Data Analysis, Belmont, CA. Wadsworth, (1983).

[11] Chen, G. and Balakrishnan, N., (1995). A General Purpose Approximate Goodness-of-fit Test. Journal of Quality Technology, 27, 154-161.

[12] Cooray, K. and Ananda, M. (2008). A Generalization of the Half-Normal Distribution with Application to Lifetime Data. Communications in Statistics-Theory and Methods, 37, 1323-1337.

[13] Cordeiro, G. M., Pescim, R. R., Demétrio, C. G. B. and Ortega, E. M. M. (2014). The Kummer Generalized Gamma Distribution. Journal of Data Science, 12(4), 661-698.

[14] Cordeiro, G. M., Silva, G. O., Ortega, E. M. M. (2011). The Beta-Weibull Geometric Distribution. Statistics DOI: 10.1080/02331888.2011.577897.

[15] Cordeiro, G. M., Silva, G. O., Pescim, R. R., and Ortega, E. M. M. (2014). General Properties for the Beta Extended Half-Normal Model. Journal of Statistical Computation and Simulation, 84, 881-901.

[16] Cordeiro, G. M., Ortega, E. M. M., and Popovic, B. V. (2014). The Gamma Linear Failure Rate Distribution. Journal of Statistical Computation and Simulation, 84(11), 2408-2426.

[17] Cordeiro, G. M., Ortega, E. M. M., and Lamonte, A. J. (2015). The Poisson Generalized Linear Failure Rate Model. Communications in Statistics-Theory and Methods, 44(10), 2037-2058.

[18] Cordeiro, G. M., Silva, G. O., Ortega, E. E. M., Cintra, L. C., Rago, L. C. (2011). The Beta-Weibull Geometric Distribution. Statistics: A Journal of Theoretical and Applied Statistics, 0, 1-18, DOI: 10.1080/02331888.2011.577897.

[19] Eugene, N., Lee, C., and Famoye, F. (2002). Beta-Normal Distribution and Its Applications. Communications in Statistics-Theory and Methods, 31(4), 497-512.

[20] Gradshteyn, I. S. and Ryzhik, I. M. (2000). Table of Integrals, Series, and Products (sixth edition). San Diego: Academic Press.

[21] Gupta, R. D., and Kundu, D. (1999). Generalized Exponential Distribution. Australian and New Zealand Journal of Statistics, 41(2), 173-188.

[22] Jafari, A., and Mahmoudi, E. (2012). Beta-Linear Failure Rate Distribution and its Applications. arXiv:1212.5615v1 [stat.ME]. 
[23] Khan, M. S. (2010). The Beta Inverse Weibull Distribution. International Transactions in Mathematical Sciences and Computer, 3, 113-119.

[24] Lee, C., Famoye, F., Olumolade, O. (2007). Beta-Weibull Distribution: Some Properties and Applications to Censored Data. Journal of Modern Applied Statistical Methods, 6, 173-186.

[25] Mahmoudi, E., and Jafari, A. (2014). The Compound Class of Linear Failure Rate-Power Series Distributions: Model, Properties and Applications. arXiv:1402.5282v1 [stat.CO].

[26] Marshall, A. W., and Olkin, I. (2007). Life Distributions: Structure of Nonparametric, Semi parametric and Parametric Families. New York: Springer.

[27] Mudholkar, G. S., Srivastava, D. K. and Freimer, M. (1995). The Exponentiated Weibull Family: A Reanalysis of the Bus-Motor Failure Data. Technometrics, 37, 436-445.

[28] Nadarajah, S., and Kotz, S. (2004). The Beta Gumbel Distribution. Mathematical Problems in Engineering, 4, 323-332.

[29] Nadarajah, S., and Kotz, S. (2006). The Beta Exponential Distribution. Reliability Engineering and System Safety, 91, 689-697.

[30] Oluyede, B. O., Mutiso, F., and Huang, S. (2015). The Log Generalized Lindley-Weibull Distribution with Application. Journal of Data Science, 13(2), 281-310.

[31] Oluyede, B. O., and Yang, T. (2015). A New Class of Generalized Lindley Distribution with Applications. Journal of Statistical Computation and Simulations, 85(10), 2072-2100.

[32] Pal, M. Masson, A. M., Woo, J. (2006). On the Exponentiated Weibull Distribution. Statistica, 66, 139-147.

[33] Percontini, A., Blas, B., and Cordeiro, G. M. (2013). The Beta Weibull Poisson Distribution. Chilean Journal of Statistics, 4(2): 3-26.

[34] Pescim, R. R., Demétrio, C.G.B., Cordeiro, G. M., Ortega, E. M. M., and Urbano, M. R. (2010). The Beta Generalized Half-Normal Distribution. Computational Statistics and Data Analysis, 54, 945-957.

[35] Paranaíba, P. F., Ortega, E. M. M., Cordeiro, G. M., and Pescim, R. R. (2011). The Beta Burr XII Distribution with Applications to Lifetime Data. Computational Statistics and Data Analysis, 55, 1118-1136.

[36] Proschan, F. (1963). Theoretical Explanation of Observed Decreasing Failure Rates. Technometrics, 5, 375-383. 
[37] Rényi, A. (1960). On Measures of Entropy and Information. Proceedings of the Fourth Berkeley Symposium on Mathematical Statistics and Probability, 1, 547-561.

[38] Sarhan, M., and Kundu, D. (2009). Generalized Linear Failure Rate Distribution. Communications in Statistics-Theory and Methods, 38(5), 642-660.

[39] Sepanski, J. H., and Kong, L. A. (2007). Family of Generalized Beta Distributions for Income, /arXiv:0710.4614v1S [stat. ME].

[40] Silva, G. O., Ortega, E. M. M., and Cordeiro, G. M. (2010a). The Beta Modified Weibull distribution. Lifetime Data Analysis, 16, 409-430.

Received June 25, 2014; accepted September 28, 2014.

Broderick O. Oluyede

Department of Mathematical Sciences

Georgia Southern University, GA 30460, USA

boluyede@georgiasouthern.edu

Ibrahim Elbatal

Department of Mathematics and Statistics

Al Imam Mohammad Ibn Saud Islamic University, Saudi Arabia

iielbatal@imamu.edu.sa

Shujiao Huang

Department of Mathematics

University of Houston, Houston, TX 77204-3008, USA

zsh05400@math.uh.edu 
